\title{
Alternate Heegaard genus bounds distance
}

\author{
MARTIN SCHARLEMANN \\ MAGGY TOMOVA
}

\begin{abstract}
Suppose $M$ is a compact orientable irreducible 3-manifold with Heegaard splitting surfaces $P$ and $Q$. Then either $Q$ is isotopic to a possibly stabilized or boundarystabilized copy of $P$ or the distance $d(P) \leq 2 \operatorname{genus}(Q)$.

More generally, if $P$ and $Q$ are bicompressible but weakly incompressible connected closed separating surfaces in $M$ then either

- $\quad P$ and $Q$ can be well-separated or

- $P$ and $Q$ are isotopic or

- $d(P) \leq 2 \operatorname{genus}(Q)$.

57N10; 57M50
\end{abstract}

\section{Introduction}

Suppose $M$ is an irreducible compact orientable 3 -manifold and $P \subset M$ is a closed connected separating surface properly embedded in $M . P$ is bicompressible if it compresses into both complementary components $A$ and $B . P$ is strongly compressible if there are compressing disks for $P$ in $A$ and $B$ which have disjoint boundaries in $P$. If this is not the case, then $P$ is weakly incompressible.

Given a closed bicompressible surface $P$ in $M$, there is a natural generalization, essentially due to Hempel [5], of the notion of strong compressibility. Let $\mathcal{U}, \mathcal{V}$ be the sets of isotopy classes of essential simple closed curves in $P$ that bound disks in $A$ and $B$ respectively. The distance $d(P)$ is the distance in the curve complex of $P$ between the set of vertices corresponding to $\mathcal{U}$ and the set of vertices corresponding to $\mathcal{V}$. Put another way, $d(P)$ is the smallest number $n \geq 0$ so that there is a sequence of essential simple closed curves $\alpha_{0}, \ldots, \alpha_{n}$ in $P$ with $\alpha_{0}$ bounding a disk in $A, \alpha_{n}$ bounding a disk in $B$ and for each $1 \leq i \leq n, \alpha_{i-1}$ and $\alpha_{i}$ can be isotoped in $P$ to be disjoint. Note that $P$ is strongly compressible if and only if $d(P) \leq 1$.

Suppose $Q$ is another closed bicompressible surface in $M$. Can $Q$ tell us anything about $d(P)$ ? Obviously it need not: If $Q$ is isotopic to $P$ then it can provide no new information about $d(P)$. At the other extreme, if $Q$ can be isotoped well away from 
$P$ (for example, if it is an unknotted handlebody inside a ball) then it also can tell us nothing about $P$. Our intention is to show that, aside from these two possibilities, in fact $d(P) \leq 2$ genus $(Q)$. In particular this conclusion follows when $P$ and $Q$ are non-isotopic irreducible Heegaard splittings for $M$. In the context of the theory of Heegaard splittings, our central results are these:

Corollary 4.5 Suppose $P$ and $Q$ are Heegaard splitting surfaces for the compact orientable 3-manifold $M$. Then either $d(P) \leq 2$ genus $(Q)$ or $Q$ is isotopic to $P$ or to a stabilization or boundary-stabilization of $P$.

One can frame the Corollary in a different way:

Corollary If a compact orientable 3-manifold $M$ has a has a genus $g$ Heegaard splitting surface $P$ with $d(P)>2 g(P)$, then

- $P$ is a minimal genus Heegaard splitting surface of $M$;

- any other Heegaard splitting surface of the same genus is isotopic to $P$; in fact,

- any Heegaard splitting surface $Q$ with genus $(Q) \leq d(P) / 2$ is isotopic to a stabilization or boundary stabilization of $P$.

We would like to thank Abby Thompson for pointing out the need, when $M$ has boundary, to include the possibility that $Q$ is a boundary-stabilized copy of $P$.

Following Kobayashi [6], Hempel [5, Theorem 2.7] describes a method to construct closed 3-manifolds that have Heegaard splittings of any specified genus $g \geq 2$ and arbitrarily large distance. Then:

Corollary For any genus $g \geq 2$ and any $k$ there is a closed 3-manifold $M$ so that

- $M$ has a genus $g$ Heegaard splitting $M=H_{1} \cup_{P} H_{2}$ and

- for each $h \leq k$ any genus $h$ Heegaard splitting of $M$ is isotopic to $P$ or to a stabilization of $P$.

Of course the second conclusion implies in particular that there are no Heegaard splittings of genus less than $g$, so we may as well take $k \geq g$.

Proof Following [5, Theorem 2.7] construct a closed 3-manifold $M$ and a Heegaard splitting $M=H_{1} \cup_{P} H_{2}$ so that genus $(P)=g$ and $d(P)>2 k$. Suppose $Q$ is a Heegaard splitting surface that is not isotopic to $P$ or to a stabilization of $P$. Then by Corollary $4.52 k<d(P) \leq 2 \operatorname{genus}(Q)$ so genus $(Q)>k$.

This work was partially supported by National Science Foundation grant DMS-0405712. 


\section{Preliminaries}

Throughout the paper, all 3-manifolds will be compact and orientable. Many of the technical foundations for the argument here were laid in Scharlemann [9], but the setting was somewhat different. In [9] there was only one bicompressible surface, it was not necessarily connected and each of its components had boundary. In order to incorporate those results we briefly review some of the relevant definitions and results there.

Definition 2.1 Let $(Q, \partial Q) \subset(M, \partial M)$ be a properly embedded orientable surface in the irreducible 3-manifold $M$. $Q$ will be called a splitting surface if no component is closed, no component is a disk, and $M$ is the union of two 3-manifolds $X$ and $Y$ along $Q$.

We abbreviate by saying that $Q$ splits $M$ into the submanifolds $X$ and $Y$.

Definition 2.2 Suppose that $(Q, \partial Q) \subset(M, \partial M)$ is either a splitting surface that splits $M$ into submanifolds $X$ and $Y$ or $Q$ is a closed connected separating surface with complementary components $X$ and $Y$. Then $Q$ is bicompressible if both $X$ and $Y$ contain compressing disks for $Q$ in $M ; Q$ is strongly compressible if there are such disks whose boundaries are disjoint in $Q$. If $Q$ is not strongly compressible then it is weakly incompressible.

Let $S_{0}, S_{1}$ be two connected compact subsurfaces in the same component $N$ of $\partial M$, with each component of $\partial S_{i}, i=0,1$ essential in $\partial M$ and $S_{0} \subset$ interior $\left(S_{1}\right)$. Let $T_{i}, i=0,1$ be the properly embedded surface in $M$ obtained by pushing $S_{i}$, rel $\partial S_{i}$, into the interior of $M$, so the region $R$ lying between $T_{0}$ and $T_{1}$ is naturally homeomorphic to $S_{1} \times I$. (The boundary of $R$ is the union of $T_{0}, T_{1}$ and $S_{1}-S_{0}$.)

Definition 2.3 The properly embedded surface $T_{0} \cup T_{1} \subset M$ is called the recessed collar determined by $S_{0} \subset S_{1}$ bounding $R$. The properly embedded surface obtained by tubing $T_{0}$ to $T_{1}$ along an $I$-fiber of $S_{1} \times I \cong R$ that is incident to $T_{0}$ is called a tube-spanned recessed collar in $M$.

Proposition 2.4 [9, Proposition 2.5] Suppose $M$ is an irreducible 3-manifold, $N$ is a compressible component of $\partial M$ and $(Q, \partial Q) \subset(M, \partial M)$ is a properly embedded essential surface containing no disk components and with at least one essential component incident to $N$. Let $\mathcal{V}$ be the set of essential curves in $N$ that bound disks in $M$ and let $q$ be any component of $\partial Q$. Then, either $d(\mathcal{V}, q) \leq 1-\chi(Q)$ or $q$ lies in the boundary of a $\partial$-parallel annulus component of $Q$. 
Theorem 2.5 [9, Theorem 5.4] Suppose $M$ is an irreducible 3-manifold, $N$ is a compressible boundary component of $M$ and $(Q, \partial Q) \subset(M, \partial M)$ is a bicompressible, weakly incompressible splitting surface with a bicompressible component incident to $N$.

Let $\mathcal{V}$ be the set of essential curves in $N$ that bound disks in $M$ and let $q$ be any component of $\partial Q \cap N$. Then either

- $d(q, \mathcal{V}) \leq 1-\chi(Q)$ in the curve complex on $N$ or

- $q$ lies in the boundary of a $\partial$-parallel annulus component of $Q$ or

- one component of $Q$ is a tube-spanned recessed collar; all other components of $Q$ incident to $N$ are incompressible and $\partial$-parallel.

In addition, we note the following more technical lemma:

Lemma 2.6 Suppose $S$ is a closed connected bicompressible separating surface in $M$, dividing $M$ into components $A$ and $B$. Let $\mathcal{U}$ and $\mathcal{V}$ be the collections of simple closed curves in $S$ that bound disks on sides $A$ and $B$ respectively. Let $F \subset M$ be a properly embedded connected surface transverse to $S$. Let $F_{A}=F \cap A, F_{B}=F \cap B$ and suppose:

- All curves of $F \cap S$ are essential on $S$ and $F$.

- There is at least one $a \in \partial F_{A}$ such that $d(a, \mathcal{U}) \leq 1-\chi\left(F_{A}\right)$ and any $a \in \partial F_{A}$ for which the inequality does not hold is the boundary of an annulus component of $F_{A}$ which is parallel into $S$.

- There is at least one $b \in \partial F_{B}$ such that $d(b, \mathcal{V}) \leq 1-\chi\left(F_{B}\right)$ and any $b \in \partial F_{B}$ for which the inequality does not hold is the boundary of an annulus component of $F_{B}$ which is parallel into $S$.

Then $d(S) \leq 2-\chi(F)$.

Proof Call a component $c$ of $F \cap S A$-conforming (resp B-conforming) if $d(c, \mathcal{U}) \leq$ $1-\chi\left(F_{A}\right)\left(\operatorname{resp} d(b, \mathcal{V}) \leq 1-\chi\left(F_{B}\right)\right)$. By hypothesis there are both A-conforming components of $F \cap S$ and B-conforming components. If there is a component $c$ that is both A-conforming and $\mathrm{B}$-conforming, then

$$
d(S)=d(\mathcal{U}, \mathcal{V}) \leq d(c, \mathcal{U})+d(c, \mathcal{V}) \leq 2-\chi\left(F_{A}\right)-\chi\left(F_{B}\right)=2-\chi(F)
$$

as required.

If there is no such component, let $\gamma$ be a path in $F$ from an A-conforming component to a B-conforming component, chosen to intersect $S$ as little as possible. In particular, 
any component of $F \cap S$ incident to the interior of $\gamma$ is neither A-conforming nor B-conforming, so each of these components of $F_{A}$ and $F_{B}$ is an annulus, parallel to an annulus in $S$. It follows that the components of $F \cap S$ at the ends of $\gamma$ are isotopic in $S$. Letting $c$ be a simple closed curve in that isotopy class in $S$ we have as above

$$
d(S)=d(\mathcal{U}, \mathcal{V}) \leq d(c, \mathcal{U})+d(c, \mathcal{V}) \leq 2-\chi\left(F_{A}\right)-\chi\left(F_{B}\right)=2-\chi(F)
$$

as required.

Corollary 2.7 Suppose $M, S, A, B, \mathcal{U}$ and $\mathcal{V}$ are as in Lemma 2.6. Let $F \subset M$ be a properly embedded connected surface transverse to $S$ so that all curves of $F \cap S$ are essential on $S$ and $F$. If $F_{A}=F \cap A$ and $F_{B}=F \cap B$ are essential in $A$ and $B$ respectively, then $d(S) \leq 2-\chi(F)$.

Proof Proposition 2.4 shows that $F_{A}$ and $F_{B}$ satisfy respectively the second and third conditions of Lemma 2.6.

\section{Pairs of bicompressible surfaces and their intersection con- figurations}

Throughout this section, $P$ and $Q$ will be closed connected orientable bicompressible and weakly incompressible separating surfaces in the irreducible $\partial$-irreducible $3-$ manifold $M$. The complementary components of $P$ will be denoted $A$ and $B$; those of $Q$ will be denoted $X$ and $Y$.

We introduce the following notation: Let $P^{A}$ denote the possibly disconnected surface obtained by maximally compressing $P$ towards $A$. If a $2-$ sphere arises and the $3-$ ball it bounds in $M$ lies on the $A$ side, delete the 2-sphere. That is, add the 3-ball to the part of $M$ that lies between $P$ and $P^{A}$. In the end, $P^{A}$ may be empty; this is equivalent to $A$ being a handlebody. Similarly define $P^{B}$ by maximally compressing $P$ towards $B, Q^{X}$ by maximally compressing $Q$ towards $X$ and $Q^{Y}$ by maximally compressing $Q$ towards $Y$.

The surface $P^{A}$ separates $M$. Let $A_{-} \subset A$ denote the closure of the region that lies between $P$ and $P^{A}$ and similarly define $B_{-}, X_{-}, Y_{-}$. There is a collection of 1-handles in $A_{-}$, dual to the 2 -handles by which $P$ was compressed to $P^{A}$ so that when 1 -surgery is done to $P^{A}$ along these handles, $P$ is recovered. Alternatively, we can think of the cores of these 1 -handles as forming a graph $G$ in $A_{-}$, disjoint from $P^{A}$ except precisely on its valence one vertices. A regular neighborhood of $G \cup P^{A}$ then has boundary isotopic to the union of $P^{A}$ and $P$. We will denote $G \cup P^{A}$ by 
$\Sigma^{A}$ and call it a spine of $A_{-}$. There are parallel constructions of 3-manifolds in $M$ denoted $B_{-}, X_{-}, Y_{-}$and their spines $\Sigma^{B}, \Sigma^{X}, \Sigma^{Y}$ respectively. Notice that spines are not well-defined as graphs, but may be altered by sliding edges along edges or along the surface (eg $P^{A}$ ) to which the edge is attached.

The surfaces $P$ and $Q$ in $M$ are said to be well-separated if they can be isotoped so that $A_{-} \cup B_{-}$and $X_{-} \cup Y_{-}$are disjoint. We are interested in understanding $P \cap Q$ when these surfaces are not well-separated, so we henceforth assume this. In particular, we assume that neither $P$ nor $Q$ lies in a 3-cell, though we note below that this possibility is ruled out anyway by the assumption that each is both bicompressible and weakly incompressible.

Note that if $P^{A}$ contains a sphere component, then the ball the sphere bounds can't lie entirely in $A$ (else it would have been removed) so in fact all of $A_{-} \cup B_{-}$lies in the ball. This would mean in particular that $P$ and $Q$ are well-separated. So henceforth we further assume that no component of $P^{A}, P^{B}, Q^{X}$, or $Q^{Y}$ is a sphere. It follows that the only way in which $P$ or $Q$ could be a torus is if $M$ is a Lens space (or $S^{3}$ ) and $P$ and $Q$ are both genus one Heegaard surfaces for $M$. In that case, it is known that $P$ and $Q$ are isotopic (Bonahon-Otal [1]), so we further assume that neither $P$ nor $Q$ are tori.

Summarizing:

Henceforth assume that $P$ and $Q$ are not well-separated, that neither surface is a torus, and that no component of $P^{A}, P^{B}, Q^{X}$, or $Q^{Y}$ is a sphere.

Each of the surfaces $P^{A}, P^{B}, Q^{X}$, or $Q^{Y}$ is incompressible. For suppose, say, that $P^{A}$ or $P^{B}$ is compressible. Then $P^{A} \cup P^{B}$ is compressible. The compressing disk cannot lie in $A-A_{-}$else $P^{A}$ could be further compressed into $A$. Nor can it lie in $B-B_{-}$. Hence it lies in $A_{-} \cup B_{-}$, a manifold that is Heegaard split by $P$. Then it is a classical result, essentially due to Haken (cf Casson-Gordon [2]) that it is a weakly reducible splitting, ie $P$ is strongly compressible.

Even if we allowed $P$ and $Q$ to be well-separated, this observation rules out the possibility that $P$ or $Q$ lies in a 3-cell $C$ in $M$. For if, say, $P \subset C$ then all compressions in the construction could be done in $C$ and, ultimately, $P^{A}, P^{B}$ would, absurdly, be incompressible surfaces in the 3-cell $C$.

Suppose that $P$ and $Q$ are in general position and there are curves of intersection that are inessential in both surfaces. Each such curve $c$ bounds both a disk $D \subset P$ and a disk $E \subset Q$. If $D$ intersects $Q$ and $E$ intersects $P$ only in curves that are inessential 
in $Q$ (resp $P$ ) we say that $c$ is removable. The term reflects the fact that all such curves can be removed by isotopies of $P$ whose support lies away from any curves of intersection that are essential either in $P$ or in $Q$. Indeed, if $c$ is removable, then any component of $D \cap E$ is clearly also removable. An innermost one in $D$ (say) cuts off a disk $D^{\prime}$ from $D$ and a disjoint disk $E^{\prime}$ from $E$; neither intersects $P$ or $Q$ in essential curves. Since $M$ is irreducible, the union of $D^{\prime}$ and $E^{\prime}$ bounds a ball in $M$, which can be used to isotope $E^{\prime}$ past $D^{\prime}$, removing only removable curves. Continue the process until all removable curves are gone.

Having removed all removable curves of intersection, proceed to associate one or more of the following labels to the configuration given by $P$ and $Q$ :

- Label $A($ resp $B)$ if some component of $Q \cap P$ is a meridian circle for $A$ (resp $B)$.

- $X(\operatorname{resp} Y)$ if there is a meridian disk for $X(\operatorname{resp} Y)$ that is disjoint from $P$.

- $x($ resp $y)$ if some spine $\Sigma^{A}$ or $\Sigma^{B}$ lies entirely in $Y(\operatorname{resp} X)$.

The following is immediate:

Lemma 3.1 If the configuration of $P$ and $Q$ has no label above, then $d(P) \leq$ $2-\chi(Q)$.

Proof Consider the curves $P \cap Q$ and suppose some are essential in $P$ but inessential in $Q$. An innermost such curve in $Q$ will bound a disk in $A$ or $B$ (cf RobinsteinScharlemann [8][Lemma 4.3]). Since there is no label, such curves can not exist. In particular, any intersection curve that is inessential in $Q$ is inessential in $P$. Now suppose there is a curve of intersection that is inessential in $P$. An innermost such curve $c$ bounds a disk $D \subset P$ that lies either in $X$ or in $Y$ but, because there is no label $X$ or $Y$, this curve must be inessential in $Q$ as well. Let $E$ be the disk it bounds there. We have just seen that all intersections of $E$ with $P$ must be inessential in both surfaces, so $c$ is removable and would have been removed at the onset. We conclude that all remaining curves of intersection are essential in both surfaces.

If $P \cap Q=\varnothing$ then $Q \subset A$ say so $B$ is entirely contained in $X$ or in $Y$, say in $Y$. But $B$ contains all spines $\Sigma^{B}$ so there will be a label $x$ contradicting the hypothesis. Thus $P \cap Q \neq \varnothing$.

If $Q_{A}=Q \cap A$ were compressible in $A$ the boundary of the compression disk would also be essential in $Q$, since no curve of $Q \cap P$ is inessential in $Q$. In other words, there would be a meridian disk for $X$ or $Y$ contained in $A$. As there are no labels $X$ or $Y, Q_{A}$ and similarly $Q_{B}=Q \cap B$ must be incompressible in $A$ and $B$ respectively. 
Now suppose that every component of $Q_{A}$ were parallel into $P$ so the region between each component of $Q_{A}$ and $P$ is a product. We could then use this product structure to isotope $Q_{A}$ so that it is disjoint from a spine $\Sigma^{A}$ contradicting that the region is not labeled. Therefore $Q_{A}$ and $Q_{B}$ must be essential in $A$ and $B$ respectively. The bound on the distance then follows by Corollary 2.7 .

Lemma 3.2 If there is a spine $\Sigma^{A} \subset Y$ then any meridian disk for $Y$ that is disjoint from $P$ must intersect $\Sigma^{A}$.

Proof Since $\Sigma^{A} \subset Y, Q$ is disjoint from the separating surface $P^{A}$. In particular, $Q$ either lies in $A-A_{-}$or in $B \cup A_{-}$. The former is impossible, since all compressions of $Q$ could then be done in $A-A_{-}$(since $P^{A}$ is incompressible) implying $P$ and $Q$ are well-separated. So $Q \subset B \cup A_{-}$.

Suppose $E$ is a meridian disk for $Y$ that is disjoint from $P$ and from $\Sigma^{A}$. Use the product structure between $P$ and $\Sigma^{A}$ to push all of $Q \cap A$, as well as $E$, into $B$; after that push, maximally compress $Q \cap B$ in $B \cap Y$ to get a surface $Q^{\prime}$, an incompressible surface in $B$. Compressing disks for $P$ in $B$ can be taken to be disjoint from $Q^{\prime}$, so $P^{B}$ can be chosen so that $Q^{\prime} \cap B_{-}=\varnothing$. That is, $B_{-}$remains completely in $Y$ even after $Q$ is compressed sufficiently into $Y$ to make $Q^{\prime}$. This means that $Q$ itself is disjoint from $B_{-}$. By the argument just given, this would imply again that $P$ and $Q$ can be well separated.

Of course the symmetric statements hold if $\Sigma^{A} \subset X, \Sigma^{B} \subset Y$ or $\Sigma^{B} \subset X$.

Lemma 3.3 Suppose there is a collar $P \times I$ of $P$ and $Q \subset P \times I$. Then

- if $Q$ separates the ends of $P \times I$, then $P$ and $Q$ are isotopic and

- if $Q$ fails to separate the ends of $P$ then $Q$ compresses to exactly one side in $P \times I$ and that side is a handlebody.

Proof If $Q$ is incompressible in $P \times I$ then it is isotopic to $P$ and we have the first conclusion.

Suppose then that $Q$ is compressible into $Y \cap(P \times I)$. Maximally compress into $Y \cap(P \times I)$ and cap off any resulting spheres with balls in $Y$. If the result is the empty set, then $Y$ is a handlebody completely contained in $P \times I$. If $Q$ fails to also compress in $X \cap(P \times I)$ then we have the second conclusion.

If $Q$ does compress in both $Y \cap(P \times I)$ and in $X \cap(P \times I)$ then a maximal compression in one direction or the other won't yield a handlebody, since $P \times I$ has boundary. 
So, with no loss of generality, we are reduced to the case in which the result of a maximal compression into $Y \cap(P \times I)$ is a possibly disconnected but non-empty surface $Q^{\prime} \subset(P \times I)$. We show that this leads to a contradiction.

Let $V \subset Y$ denote the corresponding compression body lying between $Q$ and $Q^{\prime}$. Then $V \cup X_{-} \subset M$ is a manifold Heegaard split by $Q$ and, since $Q$ is weakly incompressible this is a strongly irreducible splitting. It follows from [2] that $Q^{\prime}$ can't compress in this manifold; since $Q^{X}$ is incompressible in $M$, it follows that $Q^{\prime}$ doesn't compress in $V \cup X$. Hence $Q^{\prime}$ is incompressible in $P \times I$ and so consists of a number of parallel copies of $P$. Since $Q$ is connected and lies on one side of $Q^{\prime}$, the number of copies of $P$ in $Q^{\prime}$ is one if $Q$ separates the ends of $P \times I$ and two if it does not.

If $Q^{\prime}$ were a single copy of $P$ then it would compress in $M$ on both its sides, whereas we have established that $Q^{\prime}$ is incompressible on at least one side. On the other hand, suppose that $Q^{\prime}$ consists of two copies of $P$ and that $Q$ lies between them. Then $V \cup X_{-}$is a strongly irreducible Heegaard splitting of $P \times I$, in which both boundary components lie in the same compression body $V$. These are understood [10]; in particular, $X_{-}$is the handlebody obtained by removing the neighborhood of a single $I$-fiber of $P \times I$. We have established that both complementary components of $P \times I$ in $M$ lie in $Y$; one of them is homeomorphic to $A$, the other to $B$. In the end of $P \times I$ (ie, one component of $Q^{\prime}$ ) that abuts $A$, choose an essential simple closed curve $a$ that bounds a disk $E$ in $A$, hence in $Y-V$. Let $c$ be an essential simple closed curve in $P$ that intersects $a$ in at most one point. Then the annulus $(c \times I) \subset(P \times I)$ intersects $E$ in at most one point. Removing a vertical fiber from $c \times I$ turns the annulus into an essential disk $D$ in $X$ intersecting $E$ in at most one point. This leads to the contradiction that $Q$ is strongly compressible.

Lemma 3.4 If there is a configuration labeled both $x$ and $Y$ but not $A$ or $B$ (or symmetrically $X$ and $y$ but not $A$ or $B$ ) then either $P$ and $Q$ are isotopic or $d(P) \leq$ $2-\chi(Q)$.

Proof From the label $x$ we may assume, with no loss of generality, that $\Sigma^{A} \subset Y$. From the label $Y$ we know that $Q$ is compressible in $Y-P$. Following Lemma 3.2 we know that any such compression disk $E$ intersects $\Sigma^{A}$.

We first argue that we may as well assume that all components of $P \cap Q$ are essential in $P$. For suppose not; let $c$ be the boundary of an innermost disk $D$ in $P-Q$. If $c$ were essential in $Q$ then $D$ can't be in $Y$ (by Lemma 3.2) and so it would have to lie in $X$. But $D$ is disjoint from $E \subset A$, contradicting the weak incompressibility of $Q$. We deduce that $c$ is inessential in $Q$. Replace the disk that it bounds in $Q$ with $D$. This changes the configuration, but it clearly can't remove a label of the form $x, y, X, Y$ 
(though conceivably it may add such a label as the part of $Q$ removed might intersect potential spines of $A_{-}$or $B_{-}$or meridian disks for $X$ or $Y$.) So the new configuration satisfies the hypothesis also, and has at least one fewer curve of intersection. Continue until all curves of intersection that are inessential in $P$ are removed. Notice that the moves on $Q$ are all isotopies, so the process does not change the fact that $P$ and $Q$ can not be well-separated. Suppose now that some curve of intersection is inessential on $Q$. By the above it must be essential on $P$ but then an innermost such curve would give rise to a label $A$ or $B$ contradicting the hypothesis. Thus $Q \cap A$ and $Q \cap B$ have no disk components and therefore have nonpositive Euler characteristic.

By Lemma 3.2 $Q \cap A$ does not compress into $Y$ in $A-\Sigma_{A}$ so it is either incompressible there or else it compresses into $X$ there. The latter would imply that $Q \cap A$ is actually bicompressible in $A$. We show that in either case, for each component $q$ of $Q \cap P$ that is not the boundary of a $\partial$-parallel annulus in $A, d(q, \mathcal{U}) \leq 1-\chi(Q \cap A)$.

Case $1 Q \cap A$ is incompressible in $A-\Sigma_{A} \cong P \times I$.

Then each component of $Q \cap A$ must be boundary-parallel into $P$. The compression disk of $Q \cap A$ in $Y-P$ can be extended via this parallelism to give a meridian disk for $A$ that is disjoint from $Q \cap P$ and thus disjoint from all $q \in Q \cap P$. Hence $d(q, \mathcal{U}) \leq 1 \leq 1-\chi(Q \cap A)$.

Case $2 Q \cap A$ is bicompressible in $A$.

$Q \cap A$ can't be a tube-spanned recessed collar (else it would compress in $A-\Sigma_{A}$ ) so, by Theorem 2.5, $d(q, \mathcal{U}) \leq 1-\chi(Q \cap A)$ for all $q \in \partial Q$ which are not the boundary of an annulus parallel into $P$.

Now consider $Q \cap B$. It is incompressible in $B$ because a compression into $Y$ would violate Lemma 3.2 and a compression into $X$ would provide a weak reduction of $Q$. If $Q \cap B$ is essential in $B$ then by Proposition 2.4 $Q$ satisfies the hypotheses for $F$ in Corollary 2.7 and the conclusion of that Proposition completes the proof. If $Q \cap B$ is not essential in $B$ then every component of $Q \cap B$ is parallel into $P$ so $Q \subset P \times I$. It follows from Lemma 3.3 that either $Q$ is isotopic to $P$ (and we are done) or $X$ is a handlebody contained in $P \times I$.

Consider the latter possibility, $X$ is a handlebody contained in a collar of $P$. We know that some compressing disk $E$ in $Y$ lies entirely in $A$, so in particular it is disjoint from the end of $P \times I$ that lies in $B$. Put another way, we can push the handlebody $X$ into $A$ along with $E$. Once both $X$ and $E$ lie in $A$, maximally compress $Q$ into $Y \cap A$ and call the resulting surface $Q^{\prime}$. $Q^{\prime}$ is incompressible in $A$ (since $Q$ is weakly incompressible), so all compressing disks for $P$ in $A$ can be made disjoint from $Q^{\prime}$ and hence from $Q$. That is, $P^{A}$ separates $P$ from $Q$. As usual, this would imply that $P$ and $Q$ are well-separated, a contradiction to our underlying assumption. 
Lemma 3.5 If there is a configuration labelled both $X$ and $Y$ but not $A$ or $B$ then either $P$ and $Q$ are isotopic or $d(P) \leq 2-\chi(Q)$.

Proof Since $Q$ is weakly incompressible, any pair of meridian disks, one in $X$ and one in $Y$, must intersect on their boundaries and so cannot be separated by $P$. It follows that if both labels $X$ and $Y$ appear then the only meridian disks for $X$ and $Y$ that are disjoint from $P$ are both in $A$, say, so $Q_{A}=Q \cap A$ is bicompressible in $A$ and $Q_{B}=Q \cap B$ is incompressible in $B$.

Again we may as well assume that all components of $P \cap Q$ are essential in $P$. For suppose not; let $c$ be the boundary of an innermost disk $D$ in $P-Q$. If $c$ were essential in $Q$ then a disk in $B$ parallel to $D$ would provide a compression of $Q_{B}$ in $B$. From this contradiction we deduce that $c$ is inessential in $Q$ and proceed as in the proof of Lemma 3.4. As no labels $A$ or $B$ appear, all curves are also essential on $Q$ and therefor $Q \cap A$ and $Q \cap B$ have nonpositive Euler characteristic.

Following Theorem 2.5 there are two cases to consider:

Case $1 d(\mathcal{U}, q) \leq 1-\chi\left(Q_{A}\right)$ for each component $q$ of $P \cap Q$ that is not the boundary of an annulus component of $Q_{A}$ parallel into $P$.

If $Q_{B}$ is essential then this case follows much as in Lemma 3.4: by Proposition $2.4 Q$ satisfies the hypotheses for $F$ in Corollary 2.7 and the conclusion of that Proposition completes the proof. If $Q \cap B$ is not essential in $B$ then every component of $Q \cap B$ is parallel into $P$ so $Q$ would be disjoint from a spine $\Sigma^{B}$. This would imply that $\Sigma^{B}$ is contained in either $X$ or $Y$ so the region would also be labeled $x$ or $y$ and Lemma 3.4 applies.

Case $2 Q_{A}$ is the union of a tube-spanned recessed collar and some components parallel into $P$.

If $Q_{A}$ is the union of a tube-spanned recessed collar and some some components parallel into $P$, then $\Sigma^{A}$ can be made disjoint from $Q$ as $Q_{A}$ is contained in a collar of $P$. Thus Lemma 3.4 applies again.

Lemma 3.6 If there is a configuration labeled both $x$ and $y$ but not $A$ or $B$, then either $P$ and $Q$ are isotopic or $d(P) \leq 2-\chi(Q)$.

Proof As usual, we can assume that all curves in $P \cap Q$ are essential in both surfaces. Indeed, if there is a curve of intersection that is innessential in $P$ then an innermost one either is inessential also in $Q$, and can be removed as described above, or is essential in $Q$ and so would give a rise to a label $X$ or $Y$. In the latter case, the result would 
follow from Lemma 3.4. As no labels $A$ or $B$ appear, we can again assume that all curves are also essential on $Q$ and thus $Q_{A}$ and $Q_{B}$ have no disk components.

Both $X$ and $Y$ contain entire spines of $A_{-}$or $B_{-}$, though since we are not dealing with fixed spines the labels could arise if there are two distinct spines of $A_{-}$, say, one in $X$ and one in $Y$. Indeed that is the case to focus on, since if spines $\Sigma_{A} \subset X$ and $\Sigma_{B} \subset Y$ then $Q$ lies entirely in $P \times I$ and separates its ends. By Lemma 3.3 this implies that $Q$ is isotopic to $P$.

So suppose that $\Sigma_{A} \subset Y$ and another $\Sigma_{A}^{\prime} \subset X$. Suppose $P^{A} \neq \varnothing$ (ie, $A$ is not a handlebody). Then the parallel incompressible surfaces $P^{\prime A}$ and $P^{A}$ lie respectively in $X$ and $Y$. That is, $Q$ lies in a collar $P^{A} \times I$. Since $P^{A}$ is incompressible in $M$, $Q$ can be fully compressed into both $X$ and $Y$ entirely within $P^{A} \times I$. Hence $Q^{X}$ is parallel to $P^{\prime A}, Q^{Y}$ is parallel to $P^{A}$ and $Q$ is a Heegaard splitting of the product $P^{A} \times I$ and separates its ends. But by Scharlemann-Thompson [10] this would imply that $Q$ is strongly compressible, a contradiction.

So we conclude that $A$ is a handlebody. If $Q \cap A$ compresses in $X \cap A$ (resp $Y \cap A$ ) then the configuration has both labels $X$ and $y$ (resp $Y$ and $x$ ), and we are done via Lemma 3.4. So we henceforth assume that $Q \cap A$ is incompressible in $A$. Then it is certainly incompressible in the product $A-\Sigma_{A}$ and so every component of $Q \cap A$ is parallel in $A-\Sigma_{A}$ to a subsurface of $P$. Similarly every component of $Q \cap A$ is parallel in $A-\Sigma_{A}^{\prime}$ to a subsurface of $P$.

Any arc in $A$ between $\Sigma_{A} \subset Y$ and $\Sigma_{A}^{\prime} \subset X$ must intersect $Q \cap A$ an odd number of times, so there is a component $Q_{0}$ of $Q \cap A$ that such an arc intersects an odd number of times. Put another way: $Q_{0}$ is a component of $Q \cap A$ that lies between $\Sigma_{A}$ and $\Sigma_{A}^{\prime}$. This implies that $Q_{0}$ is parallel into $P$ on both its sides, ie that $A \cong Q_{0} \times I$. Since $P$ is not a torus, $Q_{0}$ is not an annulus.

Let $\alpha$ be an essential arc in $Q_{0}$. Then $\alpha \times I \subset Q_{0} \times I \cong A$ is a meridian disk $D$ for $A$ that intersects $Q_{0}$ precisely in $\alpha$. $\partial$-compressing $Q_{0}$ along one of the two disk components of $D-\alpha$ gives a curve or curves in $Q$ that are essential in $Q$ (since $Q_{0}$ is not an annulus) and are disjoint both from $\partial Q_{0}$ and the meridian $D \cup_{\alpha} D^{\prime}$. In particular, for $q_{0}$ any $\partial$-component of $Q_{0}, d\left(q_{0}, \mathcal{U}\right) \leq 2 \leq 1-\chi\left(Q_{0}\right)$. If $Q_{A}-Q_{0}$ has a non-annulus component, then $1-\chi\left(Q_{0}\right)<1-\chi\left(Q_{A}\right)$ and thus for any component $q$ in $P \cap Q, d(\mathcal{U}, q) \leq d\left(\mathcal{U}, q_{0}\right)+d\left(q, q_{0}\right) \leq 1-\chi\left(Q_{0}\right)+1 \leq 1-\chi\left(Q_{A}\right)$. Thus $Q_{A}$ always satisifies the hypothesis for $F_{A}$ in Lemma 2.6.

Now consider $Q_{B}=Q \cap B$. If $Q_{B}$ is essential, then by Proposition $2.4 Q_{B}$ satisfies the hypothesis for $F_{B}$ in Lemma 2.6 and we are done by that lemma. If $Q_{B}$ is compressible in $B$, we have labels $X$ and $y$ (or $x$ and $Y$ ) and we are done via Lemma 
3.4. Finally, if each component of $Q_{B}$ is parallel to a subsurface of $P$, then $Q$ is disjoint from a spine $\Sigma^{B}$ as well, a case we have already considered.

\section{How labels change under isotopy}

Consider how configurations and their labels change as $P$ is isotoped in $Q$. Clearly if there are no tangencies of $P$ with $Q$ during the isotopy then the curves $P \cap Q$ change only by isotopies in $P$ and $Q$, so there is no change in labels. Similarly, if there is an index 0 tangency, $P \cap Q$ changes only by the addition or deletion of a removable curve. Since all such curves are removed before labels are defined, again there is no affect on the labelling. So it suffices to consider only what can happen to the labelling when passing through a saddle tangency of $P$ with $Q$.

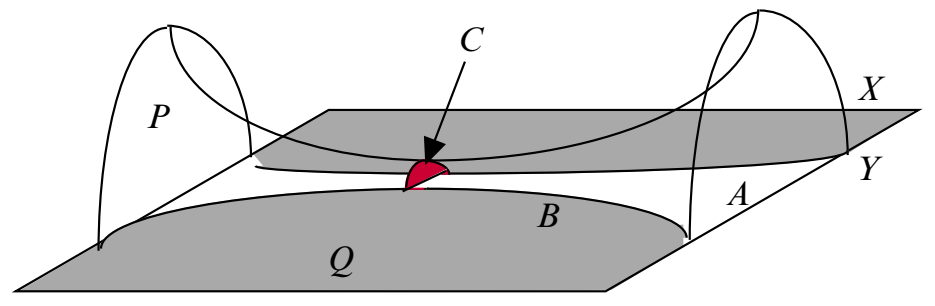

Figure 1

Lemma 4.1 Suppose a configuration is changed by passing through a saddle point, and the bigon $C$ defining the saddle tangency (cf Figure 1) lies in $X \cap A$. Then

- No label $x$ or $X$ is removed.

- No label $y$ or $Y$ is created.

- Suppose that, among the labels both before and after the move, neither $A$ nor $B$ occur. If there is no label $x$ or $X$ before the move, but one is created after and if there is a label $y$ or $Y$ before the move and none after, then either $P$ and $Q$ are isotopic or $d(P) \leq 2-\chi(Q)$.

Proof We first show that no label $x$ or $X$ is removed. If there is a meridian disk for $X$ that lies in $A$, a standard innermost disk, outermost arc argument on its intersection with $C$ shows that there is a meridian disk for $X$ in $A$ that is disjoint from $C$. The saddle move has no effect on such a disk (nor, clearly, on a meridian disk for $X$ that lies in $B$ ). If there is a spine of $A_{-}$or $B_{-}$lying entirely in $Y$ then that spine, too, is unaffected by the saddle move. 
Dually, no label $y$ or $Y$ is created: the inverse saddle move, restoring the original configuration, is via a bigon that lies in $B \cap Y$.

The third item is the most difficult to prove. A useful approach is to imagine positioning $Q$ so that it is exactly tangent to $P$ at the saddle. A bicollar of $Q$ then has ends which correspond to the position of $Q$ just before the move and just after. Let $Q_{a}$ denote $Q \cap A$ after the move and $Q_{b}$ denote $Q \cap B$ before the move. The bicollar description shows that $Q_{a}$ and $Q_{b}$ have disjoint boundaries in $P$. Moreover the complement of $Q_{a} \cup Q_{b}$ in $Q$ is a regular neighborhood of the singular component of $P \cap Q$, with Euler characteristic -1 . It follows that $\chi\left(Q_{a}\right)+\chi\left(Q_{b}\right)=\chi(Q)+1$.

With $Q$ positioned as described, tangent to $P$ at the saddle point but otherwise in general position, consider the closed (non-singular) curves of intersection.

Claim 1 It suffices to consider the case in which all non-singular curves of intersection are essential in $P$.

To prove the claim, suppose a non-singular curve is inessential and consider an innermost one. Assume first that the disk $D$ that it bounds in $P$ does not contain the singular curve $s$ (ie the component of $P \cap Q$, homeomorphic to a figure 8 , that contains the saddle point). If $\partial D$ is essential in $Q$, then it would give rise to a label $X$ or a label $Y$ that persists from before the move until after the move, contradicting the hypothesis. Suppose on the other hand that $\partial D$ is inessential in $Q$ and so bounds a disk $E \subset Q$. All curves of intersection of $E$ with $P$ must be inessential in $P$, since there is no label $A$ or $B$. It follows that $\partial D=\partial E$ is a removable component of intersection so the disk swap that replaces $E$ with a copy of $D$, removing the curve of intersection (and perhaps more such curves) has no effect on the labelling of the configuration before or after the isotopy. So the original hypotheses are still satisfied for this new configuration of $P$ and $Q$.

Suppose, on the other hand, that an innermost non-singular innesential curve in $P$ bounds a disk $D$ containing the singular component $s$. When the saddle is pushed through, the number of components in $s$ switches from one $s_{0}$ to two $s_{ \pm}$or vice versa. All three curves are innesential in $P$ since they lie in $D ; s_{0}$ and at least one of $s_{ \pm}$bounds a disk in $P$ that is disjoint from $Q$. If either is essential in $Q$ then they determine a label $X$ or $Y$ that exists both before and after the move across the saddle (since in $Q$ the curves can be isotoped away from $s$ ). This would violate the hypotheses of the third case above, so we can assume both curves, hence all three curves, are inessential in $Q$. As above, this implies that they are all removable, so passing through the singularity can have no effect at all on the labelling. This proves the claim. 
Claim 2 It suffices to consider the case in which also all three curves $s_{0}, s_{ \pm}$are essential in $P$.

The case in which all three curves are inessential in $P$ is covered in the proof of Claim 1. If two are inessential in $P$, so is the third. We're left with the case in which exactly one is inessential in $P$ and, following Claim 1, the disk it bounds in $P$ is disjoint from $Q$. If the curve were essential in $Q$ then there would have to be a label $X$ or $Y$ that occurs both before and after the saddle move, a contradiction. If the curve is inessential in $Q$ then it is removable. If this removable curve is $s_{ \pm}$then passing through the saddle can have no effect on the labelling; if this removable curve is $s_{0}$ then the curves $s_{ \pm}$are parallel in both $P$ and $Q$. In the latter case, passing through the saddle has the same effect on labelling as passing an annulus component of $P_{X}$ across a parallel annulus component $Q_{0}$ of $Q_{A}$. This move can have no effect on labels $x$ or $y$. A meridian disk $E$ for $Y$ that's disjoint from $P$ would persist after this move, unless $\partial E$ is in fact the core curve of the annulus $Q_{0}$. But then the union of $E$ and half of $Q_{0}$ would be a meridian disk of $A$ bounded by a component of $\partial Q_{0} \subset P$. In other words, there would have to have been a label $A$ before the move, a final contradiction establishing Claim 2.

Claims 1 and 2, together with the fact that neither labels $A$ nor $B$ appear, reduce us to the case in which all curves of intersection are essential in both surfaces both before and after the saddle move. Note then that $Q_{a}$ and $Q_{b}$ are incompressible in $A$ and $B$ respectively. For example, if the latter compresses in $B$ the compression must be into $Y$ (since no label $X$ exists before the move) and such a compression would persist (and so then would the label $Y$ ) after the move. Then also neither $Q_{a}$ nor $Q_{b}$ can be inessential in $A$ or $B$ respectively. For example, if all components of $Q_{b}$ are parallel into $P$ then $Q_{b}$ is disjoint from some spine of $B$ and such a spine will be unaffected by the move, resulting on the same label ( $x$ or $y$ ) arising before and after the move. We deduce that $Q_{a}$ and $Q_{b}$ are essential surfaces in $A$ and $B$ respectively.

Now apply Proposition 2.4 to both sides: Let $q_{a}$ (resp $q_{b}$ ) be a boundary component of an essential component of $Q_{a}\left(\operatorname{resp} Q_{b}\right)$. Then

$$
\begin{aligned}
d(P)= & d(\mathcal{U}, \mathcal{V}) \leq d\left(q_{a}, \mathcal{U}\right)+d\left(q_{a}, q_{b}\right)+d\left(q_{b}, \mathcal{V}\right) \\
& \leq 3-\chi\left(Q_{a}\right)-\chi\left(Q_{b}\right)=2-\chi(Q)
\end{aligned}
$$

as required.

Corollary 4.2 If two configurations are related by a single saddle move and the union of all labels for both configurations contains both $x$ or $X$ and $y$ or $Y$, but neither $A$ nor $B$ then either $P$ and $Q$ are isotopic or $d(P) \leq 2-\chi(Q)$ 
Proof With no loss, the saddle move is as described in Lemma 4.1. That lemma shows that either we are done, or there is a single configuration for which both $x$ or $X$ and $y$ or $Y$ appear. The result then follows from one of Lemmas 3.5, 3.6 or 3.4

Here is our main theorem:

Theorem 4.3 Suppose $P$ and $Q$ are bicompressible but weakly incompressible closed connected surfaces in $M$. Then either

- $P$ and $Q$ can be well-separated,

- $P$ and $Q$ are isotopic or

- $d(P) \leq 2-\chi(Q)$.

Proof We use the notation above, eg, $P$ splits $M$ into $A$ and $B$ containing spines $\Sigma^{A}$ and $\Sigma^{B}$ respectively. We will assume that $P$ and $Q$ are not well-separated.

Consider a square $I \times I$ that describes generic sweep-outs of $P$ and $Q$ from $\Sigma^{A}$ to $\Sigma^{B}$ and from $\Sigma^{X}$ to $\Sigma^{Y}$ respectively. We picture the limit of the sweep-out as $P$ gets near $\Sigma^{A}$ as represented by the left side of the square. Similarly $\Sigma^{B}$ is represented by the right side of the square, $\Sigma^{X}$ as represented by the bottom side of $I \times I$ and $\Sigma^{Y}$ as represented by the top. See Figure 2; what's in the interior of the square in that figure will now be explained.

Each point in the square represents a positioning of $P$ and $Q$. Inside the square is a graph $\Gamma$, called the graphic that represents points at which the intersection is not generic: There is a single point of tangency between $P$ and $Q$ at each point in an edge in the graphic; at each (valence four) vertex of $\Gamma$ there are two points of tangency. There are also valence two vertices corresponding to "birth-death" points, but they play no role in the discussion and we ignore them. By general position of, say, the spine $\Sigma^{A}$ with the surface $Q$ the graphic $\Gamma$ is incident to $\partial I \times I$ in only a finite number of points (corresponding to tangencies between eg $\Sigma^{A}$ and $Q$ ). Each such point in $\partial I \times I$ is incident to a single edge of $\Gamma$.

Any point in the complement of $\Gamma$ represents a generic intersection of $P$ and $Q$. Each component of the graphic complement will be called a region; any two points in the same region represent isotopic configurations, so we can label each region with labels as described above. See Figure 2. If any region is unlabelled we are done, by Lemma 3.1.

Consider what we know about the labelling near the edges of the square, that is when $P$ or $Q$ are near one of the spines. For any generic $Q, \Sigma^{A}$ is transverse to $Q$, so $Q$ 


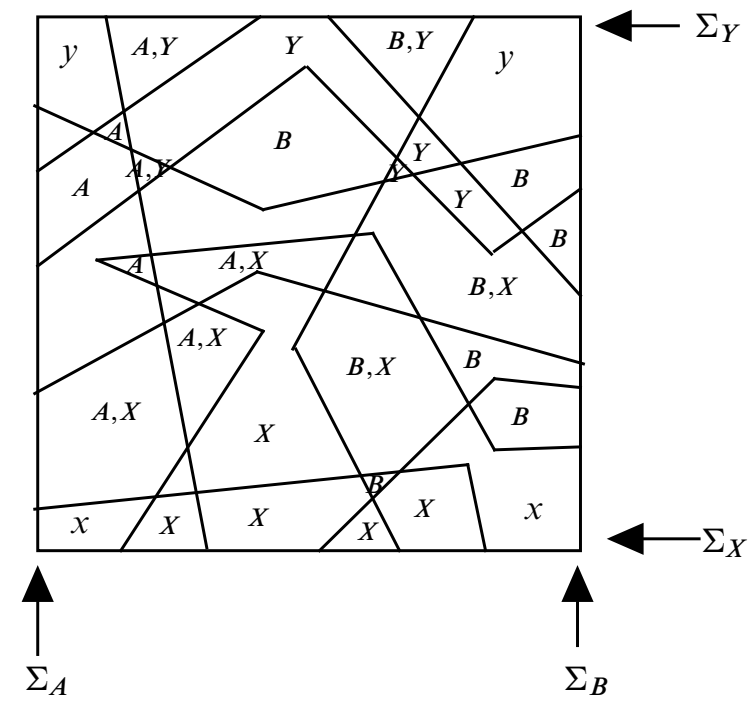

Figure 2

is either disjoint from the edges in $\Sigma^{A}$ or intersects them transversally. On the other hand, any curve in $P$ that bounds a meridian of $B$ will intersect every meridian of $A$ (since $P$ is weakly incompressible) so as $P$ limits on $\Sigma_{A}$, any meridian curve of $B$ will limit on a curve that runs multiple times along each edge in $\Sigma^{A}$. It follows that when $P$ is near $\Sigma^{A}$, and for a generic $Q$ there may or may not be a label $A$, depending on how $Q$ intersects $\Sigma^{A}$. But note that by general position all but a finite number of meridians of edges of $\Sigma^{A}$ are disjoint from $Q$. Hence in any case there is no label $B$, for such a label requires a curve of $P \cap Q$ to cross all meridians of edges of $\Sigma^{A}$.

Dually, when $P$ is near $\Sigma^{B}$ there is no label $A$.

Suppose next that $Q$ is near $\Sigma^{X}$. All but a finite number of points on the edges of $\Sigma^{X}$ will be disjoint from $P$; it follows that for $Q$ near $\Sigma^{X}$, there are meridians of $X$ that are disjoint from $P$. Hence all regions on the side of the square corresponding to $Q$ near $\Sigma^{X}$ (the bottom in the Figure) have a label $X$, as well as possibly other labels. Similarly all regions on the opposite side of the square (the top) corresponding to $Q$ near $\Sigma^{Y}$ have at least label $Y$.

Now we proceed in almost the same way as in [8, Section 5]. Let $\Lambda$ be the dual complex of $\Gamma$ in $I \times I$. Specifically, $\Lambda$ is constructed as follows: place one vertex in the interior of each face and in the interior of each edge, including edges along $\partial I \times I$ (ie components of $\partial I \times I-\Gamma$ ). Then in each face connect by an edge the vertex in 
the interior of the face with the vertex in each edge of the boundary of the face. Each vertex of $\Lambda$ lying on an interior edge $e$ of $\Gamma$ will then be incident to exactly two edges, corresponding to the faces of $\Gamma$ to which $e$ is incident. Amalgamate these two edges, removing the vertex. Then in the end, $\Lambda$ has one vertex in each face of $\Gamma$ and one vertex in each component of $\partial I \times I-\Gamma$. Each edge of $\Gamma$ not incident to $\partial I \times I$ crosses exactly one interior edge of $\Gamma$. Each component of $I \times I-\Lambda$ is called a face of $\Lambda$. Note that because each internal vertex of $\Gamma$ has valence four, each face of $\Lambda$ is a quadrilateral. That is, $\Lambda$ can be thought of as a tiling of $I \times I$ by quadrilaterals, where any two quadrilaterals are either disjoint, meet in exactly a face of each, or in a corner of each. Arbitrarily many tiles may meet at a single corner. See Figure 3.

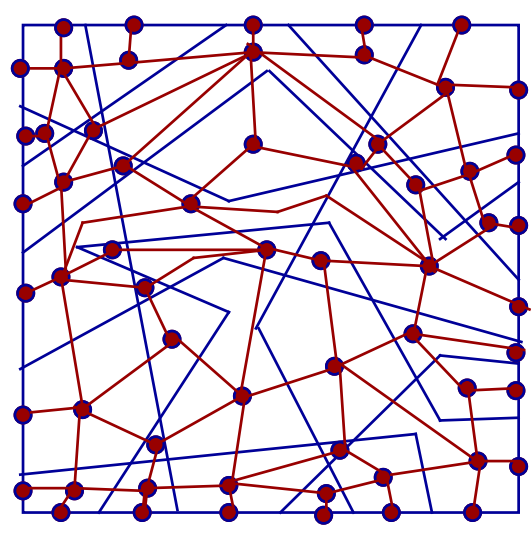

Figure 3

Now choose the following labelling scheme for each vertex in $\Lambda$ : The vertex lies in a region $R$ of $\Gamma$ which has a collection of labels. If a label $A$ or $B$ appears among the labels of $R$, associate only those labels to the vertex. If no label $A$ or $B$ appears among the labels of $R$, then associate the label $\mathrm{X}$ to the vertex if $x$ or $X$ appears among the labels of $R$ and the label $\mathrm{Y}$ to the vertex if $y$ or $Y$ appears among the labels of $R$.

The first observation is that unless $P$ is isotopic to $Q$ or $d(P) \leq 2-\chi(Q)$ (and we are done), each vertex in $\Lambda$ has exactly one label $A, B, \mathrm{X}$ or Y. Indeed, Lemma 3.1 says that every vertex has some label, and Lemmas 3.4, 3.5 and 3.6 say that no vertex of $\Lambda$ has both a label $\mathrm{X}$ and $\mathrm{Y}$. No region can have both labels $A$ and $B$ else $P$ would be strongly compressible, and no region can have both a label $A$ or $B$ and a label X or Y by our choice of labelling scheme.

Moreover, Corollary 4.2 says that vertices labelled X and Y can't even be connected by an edge in $\Lambda$. Vertices labelled $A$ and $B$ also cannot be connected by an edge 
in $\Lambda$ else $P$ would be strongly compressible. Finally, the observations above about labelling along the edges of $I \times I$ shows that no label $B$ appears along the $\Sigma^{A}$ side of $I \times I$ (the left side in the figure), no label $A$ appears along the $\Sigma^{B}$ side (the right side), no label Y appears along the $\Sigma^{X}$ side (the bottom) and no label X appears along the $\Sigma^{Y}$ side (the top).

We now appeal to the following quadrilateral variant of Sperner's Lemma:

Lemma 4.4 Suppose a square $I \times I$ is tiled by quadrilaterals so that any two that are incident meet either in a corner of each or in an entire side of each. Let $\Lambda$ denote the graph in $I \times I$ that is the union of all edges of the quadrilaterals. Suppose each vertex of $\Lambda$ is labelled $N, E, S$, or $W$ in such a way that

- no vertex on the East side of $I \times I$ is labelled $W$, no vertex on the West side is labelled $E$, no vertex on the South side is labelled $N$ and no vertex on the North side is labelled $S$.

- no edge in $\Lambda$ has ends labelled $E$ and $W$ nor ends labelled $N$ and $S$.

Then some quadrilateral contains all four labels

Two different proofs are given in a brief appendix below.

In our context the lemma says that there are four regions in the graphic incident to the same vertex of $\Gamma$ labelled $A, B, \mathrm{X}$ and $\mathrm{Y}$. (See Figure 4). Note then that only two saddle moves are needed to move from a configuration labelled $A$ to one labelled $B$. The former configuration includes a meridian circle of $A$ and the latter a meridian of $B$. If, in either the region labeled $\mathrm{X}$ or the region labeled $\mathrm{Y}$, there is a curve of intersection that is essential in $P$, then the two saddle moves show that $d(P) \leq 2 \leq 2-\chi(Q)$, as required.

Suppose then that in both regions labeled $\mathrm{X}$ and $\mathrm{Y}$ all curves of intersection are inessential in $P$. It is easy to see (cf the discussion before [8][Lemma 5.6]) that at the vertex of $\Gamma$ the two saddle tangencies lie on the same singular component of $P \cap Q$. In particular, corresponding to the region labeled $\mathrm{x}$ there is a curve in $P \cap Q$, inessential in $P$, to which separate bands are attached, one giving rise to the label $A$ and one giving rise to the label $B$. (Note that if one of these bands connected different inessential curves, the resulting curve would still be inessential in $P$ and so could not give rise to either label $A$ or $B$.) Our assumption is that attaching both labels simultaneously, corresponding to the region labeled $\mathrm{Y}$, gives rise to only inessential curves in $P$. The only way to do two simultaneous essential band moves to an inessential curve and recover only inessential curves is if the union of the two bands is the spine of a torus, ie $P$ must be a torus, contradicting our initial assumption. 


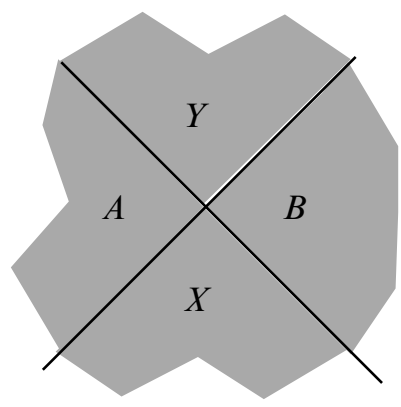

Figure 4

Corollary 4.5 Suppose $P$ and $Q$ are both Heegaard splitting surfaces for the closed orientable 3-manifold $M$. Then either $d(P) \leq 2 \operatorname{genus}(Q)$ or $Q$ is isotopic to $P$ or to a stabilization of $P$.

Proof If $P$ is weakly reducible then $d(P) \leq 1$ and we are done. Since any Heegaard splitting of a reducible 3 -manifold is reducible, we may as well assume that $M$ is irreducible and $P$ is strongly irreducible. We may as well assume $Q$ is not stabilized, so if $Q$ is weakly reducible then there is an incompressible surface $Q^{\prime}$ in $M$ with smaller genus [2]. In this case, the result follows from Hartshorn's theorem [4] (see also [9]). So we may as well assume that also $Q$ is strongly irreducible.

$P$ and $Q$ are not well-separated, for if they were there would be an incompressible closed surface lying entirely on one side of $Q$, ie inside a handlebody. Since $P$ and $Q$ are strongly irreducible Heegaard surfaces (thus weakly incompressible surfaces) and are not well-separated, the result follows immediately from Theorem 4.3.

Much the same is true when $M$ has boundary, but high distance in $P$ is also possible if $Q$ is a boundary-stabilization of $P$ (cf Moriah [7]). We briefly explain what this means: Suppose $M=A \cup_{P} B$ is a Heegaard splitting for $M$ and $N$ is a boundary component of $M$ lying in $A$. Then, since $A$ is a compression body, there is a collar $N \times I$ embedded in $A$ so that the collar is one of the components that remain when $P$ is maximally compressed into $A$. The end $N \times\{0\}$ is identified with $N \subset \partial M$ and the complement $A-(N \times I)$ is incident to the collar via 1-handles attached at $N \times\{1\}$. Consider a spanning arc $\{*\} \times I \subset N \times I$ that has one end on $P$ and the other end on $N \subset \partial M$. Add a neighborhood of $* \times[1 / 2,1]$ to the compression body $B$ and also add the subcollar $N \times[0,1 / 2]$. Symmetrically, delete the same from $A$. Call the resulting 3-manifolds $A^{\prime}$ and $B^{\prime}$ respectively.

The resulting decomposition $M=A^{\prime} \cup_{P^{\prime}} B^{\prime}$ is also a Heegaard splitting of $M$, called the boundary stabilization of $A \cup_{P} B$ along $N$. To see this, note that $A^{\prime}$ is still a 
compression body because the complement $N-\eta(*)$ of a disk in a closed surface is a 2 -dimensional handlebody, so $(N-\eta(*)) \times[1 / 2,1]$ is a 3 -dimensional handlebody.

The following Lemma, a version of the central theorem of [2] for the case of 3manifolds with boundary, was pointed out to us by Abby Thompson:

Lemma 4.6 Suppose $M=X \cup_{Q} Y$ is a genus $g$ Heegaard splitting of a 3-manifold $M$ Then either

- $Q$ is strongly irreducible

- $Q$ is stabilized

- $Q$ is boundary stabilized

- $M$ contains an essential closed surface of genus $<g$.

Proof The argument is essentially in [7]: Unless $Q$ is strongly irreducible, maximally weakly reduce the surface, producing the surface $F$. It is shown in [2] that, unless $Q$ is stabilized, $F$ is incompressible. The result of the disjoint compressions of $Q$ into $X$ and $Y$ are disks in $F$ which we imagine colored respectively red and blue. Since tubes compressed in $Q$ only connect disks of the same color, and $Q$ is connected, some component $F_{0}$ of $F$ has both red and blue disks. If $F_{0}$ is not boundary parallel then it is an essential closed surface of genus $<g$. So suppose $F_{0}$ is parallel to a boundary component via a collar $F_{0} \times I$. The original splitting is obtained by amalgamating along $F_{0}$ a splitting $Q^{\prime}$ for $M$ and a splitting on $F_{0} \times I$. Since both colors of disks appear on $F_{0}$ the splitting on $F_{0} \times I$ is non-trivial. According to the classification of splittings of $F_{0} \times I$ (cf [10]) the only non-stabilized non-trivial splitting on $F_{0} \times I$ is obtained by a single vertical tubing of collars of both its ends. Amalgamating this splitting corresponds to boundary stabilization of $Q^{\prime}$. In other words, $Q$ is the boundary stabilization of $Q^{\prime}$ along a boundary component.

Corollary 4.7 Suppose $P$ and $Q$ are both Heegaard splitting surfaces for the compact orientable 3-manifold $M$. Then either $d(P) \leq 2 \operatorname{genus}(Q)$ or $Q$ is isotopic to $P$ or to a stabilization or boundary-stabilization of $P$.

Proof Just as in the proof of Corollary 4.5 we may as well assume that $P$ is strongly irreducible and $M$ is irreducible. Following Lemma 4.6, we may as well assume that either $M$ contains an essential closed surface of genus less than genus $(Q)$ or $Q$ is strongly irreducible. In the former case, the result follows from Hartshorn's theorem [4] (see also [9]). So we are left with the case in which $Q$ is strongly irreducible. 
$P$ and $Q$ are not well-separated, for if they were there would be an incompressible closed surface, not boundary parallel, lying entirely on one side of $Q$, ie inside a compression body. As in the proof of Corollary 4.5 the result follows immediately from Theorem 4.3.

\section{Appendix: A quadrilateral Sperner's Lemma}

Here we sketch two different proofs of Lemma 4.4. The first uses algebraic topology and the second is completely elementary.

Algebraic Topology Proof Consider $\partial(I \times I)$ with its standard simplicial structure as a 4-gon. The dual complex $K$ is also a 4-gon, with a vertex in the interior of each side of $\partial(I \times I)$. There is a natural way to label the vertices of $K$ : the vertex in the East side of $\partial(I \times I)$ is labelled $E$ and so on.

Side note: Of course the identity map $\partial(I \times I) \rightarrow K$ is not a simplicial map; a useful way to think of it as a map from one 4-gon to another is shown in Figure 5.

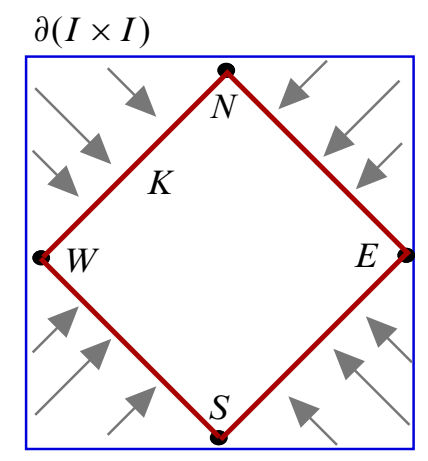

Figure 5

Now consider the subdivision of $I \times I$ given by the labelled graph $\Lambda$. We will assume that no quadrilateral face of $\Lambda$ has corners containing all 4 labels and arrive at a contradiction. This assumption means that at least two vertices of each quadrilateral have the same label. If they are diagonally opposite, add an edge between them, triangulating the quadrilateral face. If they are adjacent, add either diagonal. The resulting triangulation $\Lambda_{+}$of $I \times I$ still has the property that no edge has ends labelled $E$ and $W$ or $S$ and $N$. Map each vertex of $\Lambda$ to the vertex in $K$ with the corresponding label. Our labelling convention guarantees that any edge in $\Lambda_{+}$has 
ends labelled the same as an edge in $K$ and, moreover, no $2-$ simplex in $\Lambda_{+}$has more than two labels. It follows that the map of the vertices of $\Lambda$ extends to a simplicial map $\rho: \Lambda_{+} \rightarrow K$.

Now examine the map $\rho \mid \partial(I \times I)$. Our labelling rule guarantees that $\rho$ maps the entire East side of $I \times I$ to the two edges of $K$ that are incident to the vertex $E$ in $K$, ie to $\operatorname{star}(E)$. Similarly for the other three sides. In particular, $\rho(x)$ is never the antipode of $x$, so $\rho$ is homotopic to the identity map. But it is a classical theorem (equivalent to the Brower Fixed Point Theorem) that a map of the circle to itself that is homotopic to the identity does not extend over the disk. The contradiction completes the proof.

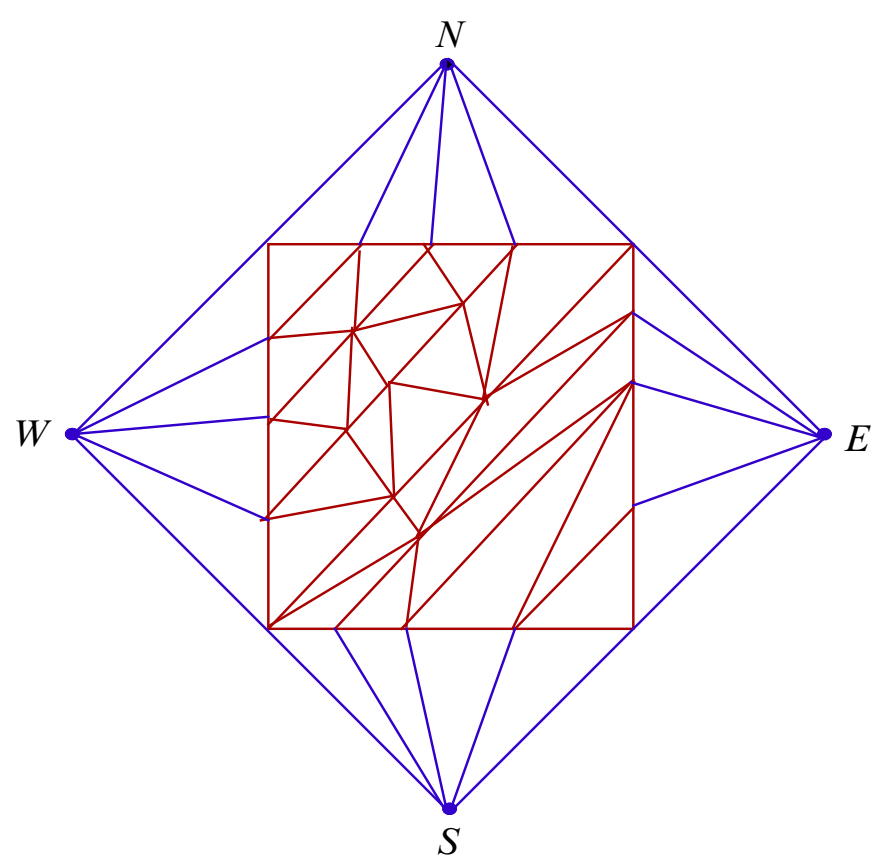

Figure 6

Elementary Proof This proof is modelled on elementary proofs of Sperner's Lemma (following very helpful comments of Francis Su, cf also [3]). As above, assume there is no such quadrilateral face, and again extend $\Lambda$ to the triangulation $\Lambda_{+}$as described in the first proof. We will give an elementary argument that there can be no triangulation of the interior of $I \times I$ that satisfies the stated labelling rules on edges and on the sides of $\partial(I \times I)$. 
Place four extra vertices in the plane outside the square, with one labelled $N$ directly north of the square, one labelled $E$ directly east, etc. Cone each of the new points to the corresponding side of the square, creating a collection of new triangles on the outside of the square. Call the union of these triangles and the original square a triangulated diamond. The diamond has four sides, each with two edges. Because no vertex on the North side of the square is labelled $S$ (and symmetrically for the other four sides) the labelling on each edge in the diamond still satisfies the given labelling rule on edges. Moreover, since the Northwest corner of the original square must have been labelled either $N$ or $W$, exactly one of the edges on the Northwest side of the diamond has exactly the labels $N$ and $W$. See Figure 6. Symmetrically, of the eight edges on the boundary of the diamond, exactly one has labels $S$ and $W$, one has labels $S$ and $E$ and one has labels $N$ and $E$. Each of the other four edges has both ends labelled the same (eg both labelled $N$ ).

The labelling rule on edges ensures that in each triangle into which the diamond is divided, at most two labels appear. Let $t$ be the number of triangles in the triangulation containing both the labels $N$ and $W$ and $e$ the number of edges in the triangulation containing both these labels. Any edge has two sides in the plane so, in the plane, there are $2 e$ sides of edges labelled exactly $N$ and $W .2 e-1$ of these sides of edges

lie inside the diamond, since, as we have seen, exactly one edge of the boundary of the diamond contains the labels $N$ and $W$. On the other hand, each triangle that contains labels $N$ and $W$ contains exactly two sides of edges labelled $N$ and $W$. Thus $2 t=2 e-1$, a parity contradiction.

\section{References}

[1] F Bonahon, J-P Otal, Scindements de Heegaard des espaces lenticulaires, Ann. Sci. École Norm. Sup. (4) 16 (1983) 451-466 (1984) MR740078

[2] A J Casson, C M Gordon, Reducing Heegaard splittings, Topology Appl. 27 (1987) 275-283 MR918537

[3] J A De Loera, E Peterson, F E Su, A polytopal generalization of Sperner's lemma, J. Combin. Theory Ser. A 100 (2002) 1-26 MR1932067

[4] K Hartshorn, Heegaard splittings of Haken manifolds have bounded distance, Pacific J. Math. 204 (2002) 61-75 MR1905192

[5] J Hempel, 3-manifolds as viewed from the curve complex, Topology 40 (2001) 631-657 MR1838999

[6] T Kobayashi, Heights of simple loops and pseudo-Anosov homeomorphisms, from: "Braids (Santa Cruz, CA, 1986)", Contemp. Math. 78, Amer. Math. Soc., Providence, RI (1988) 327-338 MR975087 
[7] Y Moriah, On boundary primitive manifolds and a theorem of Casson-Gordon, Topology Appl. 125 (2002) 571-579 MR1935173

[8] H Rubinstein, M Scharlemann, Comparing Heegaard splittings of non-Haken 3 manifolds, Topology 35 (1996) 1005-1026 MR1404921

[9] M Scharlemann, Proximity in the curve complex: boundary reduction and bicompressible surfaces, Pacific J. Math. to appear arXiv:math.GT/0410278

[10] M Scharlemann, A Thompson, Heegaard splittings of (surface) $\times I$ are standard, Math. Ann. 295 (1993) 549-564 MR1204837

Mathematics Department, University of California

Santa Barbara, CA 93106, USA

Mathematics Department, University of Iowa

Iowa City, IA 52242, USA

mgscharl@math.ucsb.edu, mtomova@math.uiowa.edu

Proposed: David Gabai

Received: 1 June 2005

Seconded: Joan Birman, Cameron Gordon

Revised: 14 November 2005 